\title{
QCD Evolution of the helicity and transversity Transverse-Momentum-Dependent parton distributions
}

\section{Alessandro Bacchetta}

Dipartimento di Fisica, Università di Pavia, and INFN Sez. di Pavia, via Bassi 6, I-27100 Pavia

E-mail: alessandro.bacchetta@unipv.it

\section{Alexei Prokudin*}

Jefferson Lab, 12000 Jefferson Avenue, Newport News, Virginia 23606, USA

E-mail: prokudinejlab.org

\begin{abstract}
We examine the QCD evolution of the helicity and transversity parton distribution functions when including also their dependence on transverse momentum. Using an appropriate definition of these polarized transverse momentum distributions (TMDs), we describe their dependence on the factorization scale and rapidity cutoff, which is essential for phenomenological applications
\end{abstract}

XXI International Workshop on Deep-Inelastic Scattering and Related Subject-DIS2013,

22-26 April 2013

Marseilles, France

\footnotetext{
*Speaker.
} 


\section{Introduction}

We present here results of calculation of ingredients of TMD Evolution of helicity and transversity TMDs. Details can be found in Ref. [1].

Our understanding of the partonic structure of hadrons relies on the study of parton distribution functions (PDFs) and their extensions. In the last years, particular attention has been devoted to transverse-momentum-dependent parton distribution functions (TMDs). Standard collinear PDFs are defined through collinear factorization theorems and obey the well-known DGLAP evolution equations $[2,3,4]$. TMDs are defined through transverse-momentum-dependent factorization and obey different evolution equations [5, 6, 7, 8, 9]. Here, for the first time we analyze these TMD evolution equations for two important distributions: the helicity and transversity TMDs.

Factorization theorems are cornerstones of our understanding of hadron structure. They describe experimentally measured cross-sections in terms of perturbatively calculable hard parts and universal structures related to nonperturbative parton dynamics, e.g., PDFs or TMDs. Factorization leads to well-defined evolution equations $[5,6,7,8,9]$ for the nonperturbative functions, which allows us to relate experimental measurements at different hard scales and perform global analyses of PDFs, TMDs, and the corresponding fragmentation functions.

The foundations of TMD factorization and evolution date back to Refs. [5, 6]. However, important details related to gauge invariance have been clarified only in the last decade (see, e.g., $[7,8,10,11,12,13])$. The first proof of TMD factorization was provided by Ji, Ma, and Yuan in Refs. $[10,8]$ while a complete definition of TMDs and rigorous proof of factorization has been recently presented by Collins in Ref. [9] and applied in Refs. [14, 15, 16, 17].

Here we consider the helicity distribution, $g_{1}\left(x, k_{T}\right)$ and the transversity distribution $h_{1}\left(x, k_{T}\right)$. They are closely related to the collinear PDFs $g_{1}(x)$ and $h_{1}(x)$, whose collinear evolution is well known (see, e.g., Refs. [18, 19, 20]).

\section{TMD Evolution}

The proper definition of TMD PDFs [9] requires the introduction of the so-called unsubtracted TMD PDFs together with further unsubtracted functions (sometimes called "soft factors"). Both of them contain rapidity divergences that are eventually canceled in the final definition of the the TMD PDFs.

The evolution of TMDs follows from their definitions, see Ref. [9]. There is a part where polarization is important. In the regime where $k_{T}$ is large compared to the hadronic scale, but still small compared to the hard scale (i.e., $\Lambda \ll\left|k_{T}\right| \ll Q$ ), TMDs can be calculated within a collinear factorization formalism $[21,22,23]$. This means that when $b_{T}$ is small but still larger than the inverse of the hard scale, i.e., $1 / Q \ll\left|b_{T}\right| \ll 1 / \Lambda$, can be written as the convultion of a perturbatively calculable hard scattering coefficient and an integrated PDF:

$$
\tilde{f}_{1}^{j}\left(x, b_{T} ; \mu, \zeta_{F}\right)=\sum_{j^{\prime}} \int_{x}^{1} \frac{d \hat{x}}{\hat{x}} \tilde{C}_{j / j^{\prime}}\left(x / \hat{x}, b_{T} ; \mu, \zeta_{F}\right) f_{1}^{j^{\prime}}(\hat{x} ; \mu)+\mathscr{O}\left(\Lambda b_{T}\right),
$$

the sum $j^{\prime}$ goes over all quark and antiquark $q$, antiquark $\bar{q}$ flavors and gluon $g$. The functions $f_{1}(\hat{x} ; \mu)$ are the ordinary integrated PDFs and the $\tilde{C}_{j / j^{\prime}}\left(x / \hat{x}, b_{T} ; \mu, \zeta_{F}\right)$ are the hard coefficient functions. Similar expressions can be written for the helicity and transversity distribution. The hard 
coefficients will be different and will be denoted by $\Delta \tilde{C}$ for helicity and $\delta \tilde{C}$ for transversity. We will explicitly calculate them for helicity and transversity distribution function.

We find the following expressions for the TMD PDF for finding a quark of flavor $j^{\prime}$ in a quark of flavor $j$ we find to order $\alpha_{s}$,

$$
\begin{array}{r}
\tilde{C}_{j / j^{\prime}}\left(x, b_{T} ; \mu ; \zeta_{F} / \mu^{2}\right)=\delta_{j^{\prime} j} \delta(1-x)+\delta_{j^{\prime} j} \frac{\alpha_{s} C_{\mathrm{F}}}{\pi}\left\{\ln \left(\frac{2 e^{-\gamma_{\mathrm{E}}}}{\mu b_{T}}\right) \frac{1+x^{2}}{(1-x)_{+}}+\frac{1}{2}(1-x)+\right. \\
\left.+\delta(1-x)\left[-\ln ^{2}\left(\frac{2 e^{-\gamma_{\mathrm{E}}}}{\mu b_{T}}\right)+\ln \left(\frac{2 e^{-\gamma_{\mathrm{E}}}}{\mu b_{T}}\right) \ln \left(\frac{\zeta_{F}}{\mu^{2}}\right)\right]\right\}+\mathscr{O}\left(\alpha_{s}^{2}\right), \\
\Delta \tilde{C}_{j / j^{\prime}}\left(x, b_{T} ; \mu ; \zeta_{F} / \mu^{2}\right)=\delta_{j^{\prime} j} \delta(1-x)+\delta_{j^{\prime} j} \frac{\alpha_{S} C_{\mathrm{F}}}{\pi}\left\{\ln \left(\frac{2 e^{-\gamma_{\mathrm{E}}}}{\mu b_{T}}\right) \frac{1+x^{2}}{(1-x)_{+}}+\frac{1}{2}(1-x)+\right. \\
\left.+\delta(1-x)\left[-\ln ^{2}\left(\frac{2 e^{-\gamma_{\mathrm{E}}}}{\mu b_{T}}\right)+\ln \left(\frac{2 e^{-\gamma_{\mathrm{E}}}}{\mu b_{T}}\right) \ln \left(\frac{\zeta_{F}}{\mu^{2}}\right)\right]\right\}+\mathscr{O}\left(\alpha_{s}^{2}\right), \\
\delta \tilde{C}_{j / j^{\prime}}\left(x, b_{T} ; \mu ; \zeta_{F} / \mu^{2}\right)=\delta_{j^{\prime} j} \delta(1-x)+\delta_{j^{\prime} j} \frac{\alpha_{S} C_{\mathrm{F}}}{\pi}\left\{\ln \left(\frac{2 e^{-\gamma_{\mathrm{E}}}}{\mu b_{T}}\right) \frac{2 x}{(1-x)_{+}}+\right. \\
\left.+\delta(1-x)\left[-\ln ^{2}\left(\frac{2 e^{-\gamma_{\mathrm{E}}}}{\mu b_{T}}\right)+\ln \left(\frac{2 e^{-\gamma_{\mathrm{E}}}}{\mu b_{T}}\right) \ln \left(\frac{\zeta_{F}}{\mu^{2}}\right)\right]\right\}+\mathscr{O}\left(\alpha_{s}^{2}\right)
\end{array}
$$

for unpolarised, helicity, and transversity TMDs respectively. The strong coupling $\alpha_{s}$ is evaluated at a scale $\mu$, and the number of active flavors is e $N_{f}$. The usual $S U\left(N_{c}\right)$ color factors are $C_{\mathrm{F}}=$ $\left(N_{c}^{2}-1\right) /\left(2 N_{c}\right), T_{f}=1 / 2$.

For the gluon contributions we obtain

$$
\begin{aligned}
\tilde{C}_{j / g}\left(x, b_{T} ; \mu, \zeta_{F} / \mu^{2}\right) & =\frac{\alpha_{s} T_{f}}{\pi}\left\{\ln \left(\frac{2 e^{-\gamma_{\mathrm{E}}}}{\mu b_{T}}\right)\left(x^{2}+(1-x)^{2}\right)+x(1-x)\right\}+\mathscr{O}\left(\alpha_{s}^{2}\right), \\
\Delta \tilde{C}_{j / g}\left(x, b_{T} ; \mu, \zeta_{F} / \mu^{2}\right) & =\frac{\alpha_{s} T_{f}}{\pi}\left\{\ln \left(\frac{2 e^{-\gamma_{\mathrm{E}}}}{\mu b_{T}}\right)(2 x-1)+(1-x)\right\}+\mathscr{O}\left(\alpha_{s}^{2}\right), \\
\delta \tilde{C}_{j / g}\left(x, b_{T} ; \mu, \zeta_{F} / \mu^{2}\right) & =0
\end{aligned}
$$

for unpolarised, helicity, and transversity TMDs, respectively. Note that there are no contributions from Soft factor at this order to quark in a gluon coefficient functions.

Eqs. $(2.3,2.4)$ and Eqs. $(2.6,2.7)$ represent the original results of this paper. They allow us to write epxressions for the helicity and transversity TMDs that fulfill TMD evolution equations and have a behavior at high transverse momentum that matches perturbative calculations. The solution for a TMD for flavor $f=q$ or $\bar{q}$ can be written in a compact way as (see Refs. [9, 14])

$$
\tilde{f}_{1}^{f}\left(x, b_{T} ; \mu, \zeta_{F}\right)=\sum_{i=q, \bar{q}, g}\left(\tilde{C}_{f / i} \otimes f_{1}^{i}\right)\left(x, b_{*} ; \mu_{b}\right) e^{\tilde{S}\left(b_{*} ; \mu_{b}, \mu, \zeta_{F}\right)} e^{g_{K}\left(b_{T}\right) \ln \frac{\sqrt{\zeta_{F}}}{\sqrt{\zeta_{f 0}}}} \hat{f}_{\mathrm{NP}}^{f}\left(x, b_{T}\right)
$$


Analogous formulas hold for the helicity, $g_{1}$, or transversity, $h_{1}$, distributions.

In order to be able to use Eq. 2.1 also at large $b_{T}$, the so-called $b_{*}$ prescription can be introduced $b_{*} \equiv b_{T} / \sqrt{1+b_{T}^{2} / b_{\max }^{2}}$.

The perturbatively calculable function $\tilde{S}\left(b_{*} ; \mu_{b}, \mu, \zeta_{F}\right)$ reads

$$
\tilde{S}\left(b_{*} ; \mu_{b}, \mu, \zeta_{F}\right)=\ln \frac{\sqrt{\zeta_{F}}}{\mu_{b}} \tilde{K}\left(b_{*} ; \mu_{b}\right)+\int_{\mu_{b}}^{\mu} \frac{d \mu^{\prime}}{\mu^{\prime}}\left[\gamma_{F}\left(g\left(\mu^{\prime}\right) ; 1\right)-\ln \frac{\sqrt{\zeta_{F}}}{\mu^{\prime}} \gamma_{K}\left(g\left(\mu^{\prime}\right)\right)\right],
$$

and expressions for $\tilde{K}, \gamma_{F}$, and $\gamma_{K}$ at order $\alpha_{S}$ can be found in Appendix B of Ref. [14].

We adopt also these other choices $\mu=Q, \mu_{b}=2 e^{-\gamma_{E}} / b_{*} \equiv b_{0} / b_{*}, \zeta_{F}=Q^{2}, \zeta_{F 0}=Q_{0}^{2}$. Different options can be explored in order to test the sensitivity of the final results to the scale choice. Note that $\tilde{K}\left(b_{*} ; \mu_{b}\right)=0$ at this order with this choice, we also set $\tilde{S}=1$ in case $\mu_{b}>\mu$ in order to avoid non physical behaviour of the Sudakov form factor.

\section{Numerical results}

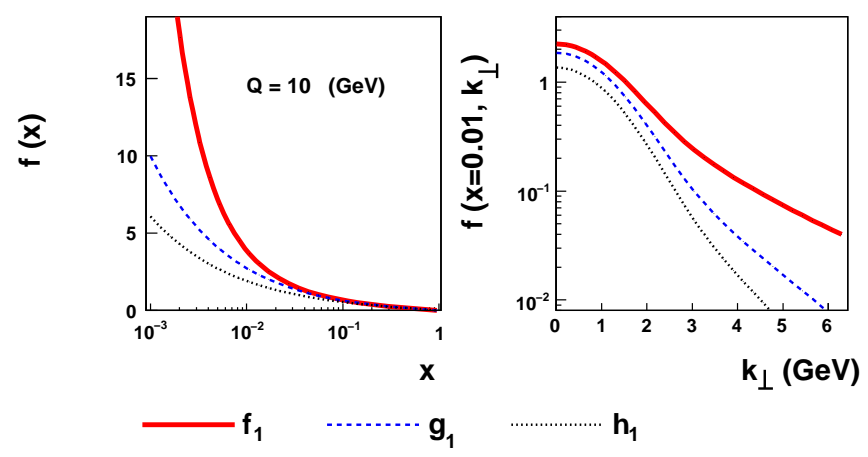

Figure 1: Evolution of $f^{u}\left(x, k_{T} ; Q, Q^{2}\right)$ and $f^{u}\left(x ; Q, Q^{2}\right)$ at two different scales $Q=10 \mathrm{GeV}$. Solid line corresponds to unpolarised evolution, dashed line corresponds to helicity evolution, and dotted line corresponds to transversity evolution.

Let us finally present results of TMD evolution using the following toy functions at initial scale $Q_{0}=1 \mathrm{GeV}: x u_{0}(x)=x d_{0}(x) \equiv x^{0.5}(1-x)^{0.5}, x \bar{u}_{0}(x)=x \bar{d}_{0}(x) \equiv 0, x g_{0}(x) \equiv x^{0.5}(1-x)^{0.5}$. Note that the choice is arbitrary as our goal is just to demonstrate the results of evolution. These initial distributions will be assumed the same for unpolarised $\left(f_{1}\right)$, helicity $\left(g_{1}\right)$, and transversity $\left(h_{1}\right)$ distributions. Results with realistic initial functions will be presented elsewhere.

The choices of non-perturbative functions that enter in Eq. 2.8 are the following (we use again the same function for all three polarization cases just for illustration purposes): $\hat{f}_{\mathrm{NP}}^{f}\left(x, b_{T}\right)=$ $\exp \left(-\frac{b_{T}^{2}\left\langle k_{T}^{2}\right\rangle}{4}\right), g_{K}\left(b_{T}\right)=-g b_{T}^{2}$, where $\left\langle k_{T}^{2}\right\rangle=0.25\left(\mathrm{GeV}^{2}\right), g=0.2\left(\mathrm{GeV}^{2}\right)$. We also choose $b_{\text {max }}=1\left(\mathrm{GeV}^{-1}\right)$.

We will show the evolution of the TMD functions choosing particular flavor $f=u$ as well as their integral over $k_{T}$ up to the value of $Q$, which we conventionally refer to as their $0^{\text {th }} k_{T}$-moment: $f^{u}\left(x, k_{T} ; \mu, \zeta_{F}\right), f^{u}\left(x ; \mu, \zeta_{F}\right) \equiv 2 \pi \int_{0}^{\mu} k_{T} d k_{T} f^{q}\left(x, k_{T} ; \mu, \zeta_{F}\right)$. Note that this $0^{\text {th }} k_{T}$-moment af a TMD functions $f^{u}\left(x ; \mu, \zeta_{F}\right)$ should not be confused with collinear PDF $f^{u}(x ; \mu)$.

In Fig. 1 we show results of the evolution of $f^{u}\left(x, k_{T} ; Q, Q^{2}\right)$ and $f^{u}\left(x ; Q, Q^{2}\right)$ at $Q=10(\mathrm{GeV})$ for the unpolarized, helicity and transversity distributions. 


\section{Conclusions}

In this paper we calculated the evolution of the transverse-momentum-dependent (TMD) helicity and transversity distribution functions. We adopted the definition of TMD PDFs as given by Collins in Ref. [9]. We provided explicit formulas for all coefficient functions at $\alpha_{S}$. The results of this paper can be readily used in TMD phenomenology.

Authored by a Jefferson Science Associate, LLC under U.S. DOE Contract No. DE-AC0506OR23177. A. Bacchetta is partially supported by the Italian MIUR through the PRIN 2008EKLACK, and by the European Community through the Research Infrastructure Integrating Activity "HadronPhysics2" (Grant Agreement n. 227431) under the $7^{\text {th }}$ Framework Programme.

\section{References}

[1] A. Bacchetta and A. Prokudin, Nucl. Phys. B, in press 2013, arXiv:1303.2129 [hep-ph].

[2] G. Altarelli and G. Parisi, Nucl. Phys., vol. B126, p. 298, 1977.

[3] Y. L. Dokshitzer, Sov. Phys. JETP, vol. 46, pp. 641-653, 1977.

[4] L. Lipatov, Sov.J.Nucl.Phys., vol. 20, pp. 94-102, 1975.

[5] J. C. Collins and D. E. Soper, Nucl. Phys., vol. B193, p. 381, 1981.

[6] J. C. Collins, D. E. Soper, and G. Sterman, Nucl. Phys., vol. B250, p. 199, 1985.

[7] X. Ji and F. Yuan, Phys. Lett., vol. B543, pp. 66-72, 2002.

[8] X. Ji, J.-P. Ma, and F. Yuan, Phys. Rev., vol. D71, p. 034005, 2005.

[9] J. Collins, Cambridge Monographs on Particle Physics, Nuclear Physics and Cosmology, Cambridge University Press, 2011.

[10] X. Ji, J.-P. Ma, and F. Yuan, Phys. Lett., vol. B597, pp. 299-308, 2004.

[11] A. V. Belitsky, X. Ji, and F. Yuan, Nucl. Phys., vol. B656, pp. 165-198, 2003.

[12] J. C. Collins, Phys.Lett., vol. B536, pp. 43-48, 2002.

[13] J. C. Collins and A. Metz, Phys. Rev. Lett., vol. 93, p. 252001, 2004.

[14] S. Aybat and T. C. Rogers, Phys. Rev., vol. D83, p. 114042, 2011.

[15] S. M. Aybat, J. C. Collins, J.-W. Qiu, and T. C. Rogers, Phys.Rev., vol. D85, p. 034043, 2012.

[16] S. M. Aybat, A. Prokudin, and T. C. Rogers, Phys.Rev.Lett., vol. 108, p. 242003, 2012.

[17] M. Anselmino, M. Boglione, and S. Melis, Phys.Rev, vol. D86 (2012) 0140282012.

[18] V. Barone, Phys.Lett., vol. B409, pp. 499-502, 1997.

[19] W. Vogelsang, Phys.Rev., vol. D57, pp. 1886-1894, 1998.

[20] A. Hayashigaki, Y. Kanazawa, and Y. Koike, Phys. Rev., vol. D56, pp. 7350-7360, 1997.

[21] J. C. Collins and D. E. Soper, Nucl. Phys., vol. B194, p. 445, 1982.

[22] X. Ji, J.-W. Qiu, W. Vogelsang, and F. Yuan, Phys. Rev. Lett., vol. 97, p. 082002, 2006.

[23] A. Bacchetta, D. Boer, M. Diehl, and P. J. Mulders, JHEP, vol. 08, p. 023, 2008. 